\title{
Reply to "Conformational fitting of a flexible oligomeric substrate does not explain the enzymatic PET degradation"
}

\author{
Hogyun Seo (i) ${ }^{1}$, In Jin Cho (i) ${ }^{2}$, Seongjoon Joo ${ }^{1}$, Hyeoncheol Francis Son ${ }^{1}$, Hye-Young Sagong ${ }^{1}$ \\ So Young Choi (iD) ${ }^{2}$, Sang Yup Lee (ib) ${ }^{2,3 \star} \&$ Kyung-Jin Kim ${ }^{1 \star}$
}

REPLYING To Wei et al. Nature Communications https://doi.org/10.1038/s41467-019-13492-9 (2019)

\section{T} he manuscript entitled "Conformational fitting of a flexible oligomeric substrate does not explain the enzymatic PET degradation" by Wei et al. ${ }^{1}$ raises a question on the conclusion reached in our published work, particularly regarding the docking calculations of PET substrate into the PETase from Ideonella sakaiensis (IsPETase) $)^{2}$. The authors showed the ethylene glycol torsion angle $\Psi$ in an amorphous PET material of $0.25 \mathrm{~mm}$ thickness (Goodfellow Cambridge Ltd.) using solid-state nuclear magnetic resonance experiments and determined trans/gauche ratio of 9:91 at $30^{\circ} \mathrm{C}$ in good agreement with the previous report on amorphous PET $(14 \pm$ $5 \%)^{3}$. Based on the result, they suggested that the conformation of the docked 2-HE(MHET) $)_{4}$ in our published work that showed a trans content of ethylene glycol higher than $25 \%$ is rarely present in amorphous PET polymer chains, and claimed that the residues in subsite IIb and IIc we suggested are unlikely to interact with the two MHET moieties of 2 -HE(MHET $)_{4}$. To support the latter statement, they further demonstrated that the transition from gauche to trans conformation is highly restricted in amorphous $\mathrm{PET}$ at $30^{\circ} \mathrm{C}$ by magic-angle-spinning nuclear magnetic resonance method. They also suggested that, instead of the perfect accommodation, the key factor facilitating the substrate binding seems to be the fragile contact between the phenylene moieties and the surrounding hydrophobic residues. In general, we agree that the comments by Wei et al. provide concrete experimental results showing that ethylene glycol units of amorphous PET polymer do not have the free rotational properties to fit into its substrate binding site as the form of $2-\mathrm{HE}(\mathrm{MHET})_{4}$ at $30^{\circ} \mathrm{C}$. Thus, the study provides the scientific community with useful information on PET degradation by IsPETase.
However, our previous docking calculations ${ }^{2}$ were independent of temperature setting and not restricted to the temperature of $30^{\circ} \mathrm{C}$. Although IsPETase cannot maintain its activity at high temperature due to its low thermal stability, efforts to increase thermal stability of the enzyme have already been reported ${ }^{4}$. Thus, an engineered IsPETase with high thermal stability might be able to accommodate the PET substrate in the manner we presented at temperatures higher than $30^{\circ} \mathrm{C}$ as the trans content of the material was increased to $56 \%$ at $70^{\circ} \mathrm{C}^{1}$.

Moreover, we did not use the Goodfellow amorphous PET in our published works 2,4 , which counters the comment that IsPETase showed almost "no" activity against crystalline PET polymer ${ }^{1}$. It was also previously shown that the semi-crystalline PET material exhibits much higher trans conformation of ethylene glycol than amorphous one at ambient temperature ${ }^{3}$. Thus, we believe that the authors' claims regarding the residues in subsite IIb and IIc may not apply under all conditions. In sum, while we agree that Wei et al. provide useful evidences that the used docking calculation is not suitable in amorphous PET at low temperature such as $30^{\circ} \mathrm{C}$, their observations are not necessarily incompatible with the general findings of our previous work ${ }^{2}$. We anticipate that Wei et al.'s work and our own will inspire future studies aimed at unraveling the exact mechanisms of PET degradation.

\section{Data availability}

Data supporting the findings of this study are available within the article and from the corresponding author upon reasonable request.

Received: 9 October 2019; Accepted: 4 November 2019;

Published online: 06 December 2019

\footnotetext{
${ }^{1}$ School of Life Sciences (KNU Creative BioResearch Group), KNU Institute for Microorganisms, Kyungpook National University, Daehak-ro 80, Buk-gu, Daegu 41566, Republic of Korea. ${ }^{2}$ Metabolic and Biomolecular Engineering National Research Laboratory, Systems Metabolic Engineering and Systems Healthcare Cross Generation Collaborative Laboratory, Department of Chemical and Biomolecular Engineering (BK21 Plus Program), Institute for the BioCentury, Korea Advanced Institute of Science and Technology (KAIST), 291 Daehak-ro, Yuseong-gu, Daejeon 34141, Republic of Korea. ${ }^{3}$ BioProcess Engineering Research Center and Biolnformatics Research Center, KAIST, 291 Daehak-ro, Yuseong-gu, Daejeon 34141, Republic of Korea. 


\section{References}

1. Wei, R. et al. Conformational fitting of a flexible oligomeric substrate does not explain the enzymatic PET degradation. Nat. Commun. (2019).

2. Joo, S. et al. Structural insight into molecular mechanism of poly(ethylene terephthalate) degradation. Nat. Commun. 9, 382 (2018).

3. Schmidt-Rohr, K., Hu, W. \& Zumbulyadis, N. Elucidation of the chain conformation in a glassy polyester, PET, by two-dimensional NMR. Science 280, 714-717 (1998).

4. Son, H. F. et al. Rational protein engineering of thermo-stable PETase from Ideonella sakaiensis for highly efficient PET degradation. ACS Catal. 9, 3519-3526 (2019).

\section{Acknowledgements}

This work was supported by the Technology Development Program to Solve Climate Changes on Systems Metabolic Engineering for Biorefineries from the Ministry of Science and ICT (MSIT) through the National Research Foundation (NRF) of Korea (NRF2012M1A2A2026556 and NRF2012M1A2A2026557).

\section{Author contributions}

H.S., S.Y.C., S.Y.L., and K.-J.K. wrote the initial paper. H.S., I.J.C., S.J., H.F.S., H.-Y.S., S.Y. C., S.Y.L., and K.-J.K. finalized the paper.

\section{Competing interests}

The authors declare no competing interests.

\section{Additional information}

Correspondence and requests for materials should be addressed to S.Y.L. or K.-J.K.

Reprints and permission information is available at http://www.nature.com/reprints

Publisher's note Springer Nature remains neutral with regard to jurisdictional claims in published maps and institutional affiliations.

\begin{abstract}
(c) (i)
Open Access This article is licensed under a Creative Commons Attribution 4.0 International License, which permits use, sharing, adaptation, distribution and reproduction in any medium or format, as long as you give appropriate credit to the original author(s) and the source, provide a link to the Creative Commons license, and indicate if changes were made. The images or other third party material in this article are included in the article's Creative Commons license, unless indicated otherwise in a credit line to the material. If material is not included in the article's Creative Commons license and your intended use is not permitted by statutory regulation or exceeds the permitted use, you will need to obtain permission directly from the copyright holder. To view a copy of this license, visit http://creativecommons.org/ licenses/by/4.0/.
\end{abstract}

(C) The Author(s) 2019 